\title{
Impedance Based Health Monitoring Technique with Probabilistic Neural Network for Possible Wall Thinning Detection of Metal Structures
}

\author{
Wongi S. $\mathrm{Na}^{1}$ and Jongdae Baek ${ }^{2}$ \\ ${ }^{1}$ Future Strategy \& Convergence Research Institute, Korea Institute of Civil Engineering \& Building Technology, \\ Gyeonggi-do 10223, Republic of Korea \\ ${ }^{2}$ Highway \& Transportation Research Institute, Korea Institute of Civil Engineering \& Building Technology, \\ Gyeonggi-do 10223, Republic of Korea \\ Correspondence should be addressed to Wongi S. Na; wongi84@naver.com
}

Received 18 July 2017; Accepted 27 August 2017; Published 9 October 2017

Academic Editor: Young-Jin Cha

Copyright (C) 2017 Wongi S. Na and Jongdae Baek. This is an open access article distributed under the Creative Commons Attribution License, which permits unrestricted use, distribution, and reproduction in any medium, provided the original work is properly cited.

\begin{abstract}
Corrosion of structures and wall thinning of pipes can severely affect the mechanical strength as wall thickness is reduced. Thus a cost effective structural health monitoring technique plays an important role when managing a structure. The electromechanical impedance (EMI) method is a local method that has limited sensing range, resulting in a high cost when covering large areas. In this study, a reattachable EMI method is investigated using a stack of multiple metal plates to conduct an experiment involving thickness reduction. In addition, the main problem of the impedance signatures changing subjected to reattaching the piezoelectric transducer is solved by using the probabilistic neural network algorithm presented for the study. The proposed approach successfully identifies the thickness of two different structures with high accuracy.
\end{abstract}

\section{Introduction}

Corrosion and wall thinning can reduce the mechanical strength of a steel structure as it can affect the metal thickness. Also, it is one of the most vital factors when dealing with life cycle costs of a structure. In this respect, nondestructive testing (NDT) plays an important role when creating an efficient health monitoring system for infrastructures. Although various NDT methods exist for damage detection, most of these methods require experts with expensive tools which can be quite heavy. To help overcome this problem, a portable monitoring system with a piezoelectric (PZT) material based device that can be attached and reattached for detecting wall thickness loss is investigated using one of the structural health monitoring techniques known as the electromechanical impedance (EMI) method. In general, a single PZT patch is attached directly to the surface of a target structure to perform the EMI method where the method was first introduced by Liang et al. [1]. In their study, the 1D model in (1) shows the coupled relationship between the electrical admittance (inverse of impedance) of the PZT element $Y(\omega)$ to the mechanical impedance of the structure $Z_{s}(\omega)$, proving that any changes in $Z_{s}(\omega)$ will affect the electrical impedance of the attached PZT element.

$$
Y(\omega)=i \omega a\left(\varepsilon_{33}^{T}(1-i \delta)-\frac{Z_{s}(\omega)}{Z_{s}(\omega)+Z_{a}(\omega)} d_{3 x}^{2} \bar{Y}_{x x}^{E}\right) .
$$

Up to date, various authors have utilized the EMI method for damage detection [2-13]. Yu et al. [2] introduced chemical corrosion using hydrochloric acid onto a pipe structure where it was detected by performing the EMI method in the frequency range of 200 to $2000 \mathrm{kHz}$ using the HP4194A impedance analyzer. The corrosion process was repeated 11 times until complete failure of the pipe surface where the changes in the real part of the impedance were evaluated using the damage index algorithm introduced in the study. Talakokula et al. [3] investigated detecting and quantifying the carbonation induced rebar corrosion level by bonding 
a PZT patch onto the surface of the rebar where the reinforced concrete specimen was exposed to carbon dioxide for over 230 days. The $\mathrm{CO}_{2}$ penetration depth was related to the equivalent stiffness parameter where the results were compared with scanning electron microscope images to show successful recognition of carbonation induced rebar corrosion in reinforced concrete. Simmers Jr. et al. [4] used an aluminum beam of $184 \mathrm{~cm}$ in size with $2.54 \mathrm{~cm}$ in width to introduce corrosion damage in 5 different places where the changes in the measured impedance signatures were quantified using the root mean square deviation (RMSD) equation. The results indicated that light corrosion can be identified up to 1.5 meters while tracking the progression of damage.

Virtually all the studies related to corrosion and damage detection using the EMI method involve permanently attaching the PZT material to a surface where the cost of the monitoring system would be high when dealing with large infrastructures. In addition, the wiring and PZT attachment on the surfaces can make the structure visually unattractive possibly raising another problem of managing the brittle PZT patch when exposed to the environment for a long time. Thus in this study, a PZT material based device that does not need to be permanently attached to a host structure is utilized [5], allowing one to easily attach and reattach to a steel structure without leaving any trail of monitoring. Due to the high sensitivity of the impedance signature, the impedance signature completely changes when reattached. This is solved by applying the probabilistic neural network (PNN) algorithm to the EMI method.

To induce the effect of wall thickness reduction for this study, multiple layers of thin metal plates of identical thickness were stacked for the experiment where removing each layer was considered as damage. With this approach, it becomes possible for one to reduce the thickness of a structure with the exact same thickness each time. Although it would be ideal to use a corrosive solution for reducing the test specimen, the EMI method is highly sensitive to small changes in the host structure and avoiding the effect of the impedance signatures changing due to the miniscule differences in the plate thickness compared to one another was considered important for this study. Thus the work presented in this study focused on the possibility of using the reattachable EMI method with the PNN technique against wall thickness reduction where this research will be continued further in a future study subjected to real corrosive environment.

\section{Electromechanical Impedance Method Setup}

For conducting the EMI method, a laptop is connected to the AD5933 evaluation board and then to the test specimen as shown in Figure 1. The evaluation board has been approved by the manufacturer (Analog Devices Inc.) of its ability to measure the real and imaginary part of the impedance up to $100 \mathrm{kHz}$ with a maximum number of 511 data points. The positive and negative sides of PZT materials are connected to the board for measuring the impedance. The equipment is fully powered by a USB cable where data is also transmitted
TAble 1: Properties of the PZT used for the study.

\begin{tabular}{lcc}
\hline Parameter & Symbol & Value \\
\hline Relative dielectric constant & $K^{T}$ & 1800 \\
Piezoelectric strain coefficient & $d_{33}$ & $390 \times 10^{-12} \mathrm{~m} / \mathrm{V}$ \\
& $d_{31}$ & $-190 \times 10^{-12} \mathrm{~m} / \mathrm{V}$ \\
Piezoelectric voltage coefficient & $g_{33}$ & $24.0 \times 10^{-3} \mathrm{~V} \mathrm{~m} / \mathrm{N}$ \\
& $g_{31}$ & $-11.6 \times 10^{-3} \mathrm{~V} \mathrm{~m} / \mathrm{N}$ \\
Coupling coefficient & $k_{33}$ & 0.72 \\
& $k_{31}$ & 0.35 \\
\hline
\end{tabular}

though this line. The PZT model of PSI-5A4E with the thickness of $0.508 \mathrm{~mm}$ was purchased (Piezo Systems Inc.) and used throughout the study where some of its properties can be seen in Table 1. Upon measuring the impedance signature, root mean square deviation (RMSD) technique is applied to analyze the data for quantifying the intensity of damage. The RMSD equation (2) shown below uses two impedance signatures in general where before $\left(Z_{k}\right)_{i}$ and after $\left(Z_{k}\right)_{j}$ damage signatures are inserted into the equation for the analysis. For this study, the real part of the impedance was used as it has been known to perform better and to be less prone to signature changes subjected to temperature variations compared to the imaginary part of the impedance [14].

$$
\operatorname{RMSD}=\left(\frac{\sum_{k=1}^{N}\left[\operatorname{Re}\left(Z_{k}\right)_{j}-\operatorname{Re}\left(Z_{k}\right)_{i}\right]^{2}}{\sum_{k=1}^{N}\left[\operatorname{Re}\left(Z_{k}\right)_{i}\right]^{2}}\right)^{1 / 2} .
$$

\section{Repeatability Performance of the Reattachable PZT Devices}

A conventional method of conducting the EMI method is to permanently attach a small piece of a PZT transducer to a surface of the target structure using a commercial adhesive. One of the issues with permanent installation is that, with large infrastructures, the cost of attaching the required number of PZT transducers for covering the target structure would be extremely high. In addition, managing such system would also be costly, as some places would be difficult for one to access (tall buildings, top of a bridge, etc.). Thus in this study, the idea of a reattachable PZT device introduced by $\mathrm{Na}$ et al. [5] was used which allows one to attach and reattach the PZT device to a steel structure without any adhesive. Figure 2 shows the reattachable device for this study where a magnet disc of $25 \mathrm{~mm}$ diameter and $5 \mathrm{~mm}$ thickness is used with the $15 \mathrm{~mm}$ square PZT transducer attached to top of its surface using a commercial adhesive (Loctite quickset epoxy). Then after 48 hours of adhesive curing, the same adhesive was used to cover the top of the PZT for protection purposes as the high frequency usage of the EMI method makes it very sensitive to signature variations. So to observe how sensitive the impedance signatures are, a simple test involving the PZT device with a square metal plate of a size $100 \mathrm{~mm}$ with $0.3 \mathrm{~mm}$ thickness was used. Here, the impedance signature 


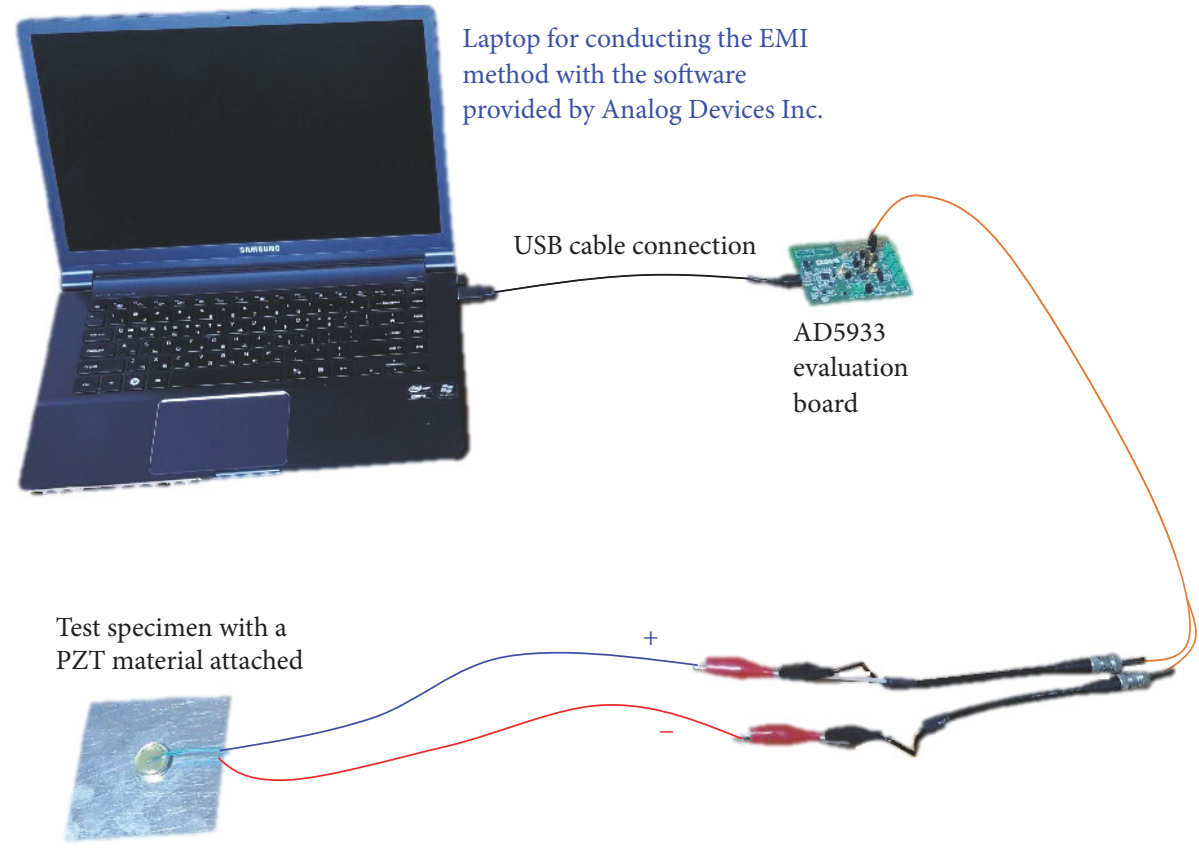

Figure 1: Photo of the AD5933 evaluation board.

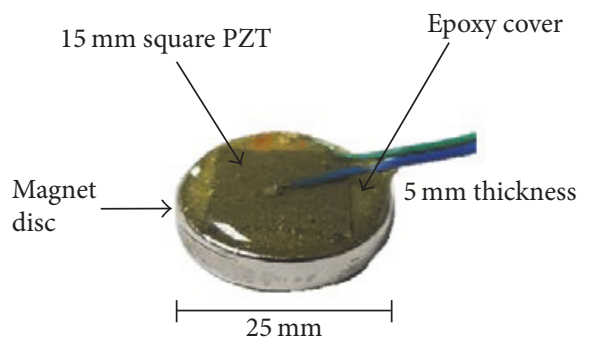

Figure 2: Reattachable PZT device.

was measured after attaching the PZT device to the plate, and then another measurement was made after detaching the PZT device. Since the PZT device was made out of a magnet, the magnetic force alone was strong enough to be attached to the metal plate without any adhesive. This process (shown in Figure 3) was repeated until 10 impedance signatures were acquired where the PZT device was attached to the center of the plate each time, visually checking its placement to simulate a real testing environment. The results of this test are plotted in Figure 4 showing how sensitive it is subjected to the reattachments. The signatures shift in either the left or right direction with the shape of the peak changing randomly. Under this circumstance, it is difficult for one to analyze if a host structure is damaged as the impedance signatures change although the actual host structure is undamaged. Thus one needs to distinguish the impedance signature changes of a damaged structure from the signature changes caused by reattaching the PZT device. To achieve this, a probabilistic neural network (PNN) algorithm was used.

\section{Application of the PNN Algorithm}

To overcome the problem of repeatability as shown in the previous section, PNN introduced by Specht [15] is used in conjunction with the RMSD equation. The training process is known to be relatively faster compared to other artificial neural network techniques and is guaranteed to converge to an optimal classifier with the increase in the size of the representative training set. One can also add or remove training samples without any extensive retraining making the PNN algorithm suitable as the integrity of a host structure can be damaged (changed) due its surrounding environment with time.

Figure 5 shows the classification algorithm with 5 categories in the pattern layer with each category representing the thickness of the host structure $(5$ metal plate layers = 5 categories) with 10 trained data. Each node in this layer contains the Gaussian function (see (3)) where the data from the input layer (500 point impedance signature data) is calculated into a single number to be processed onto the summation layer. The categories (pattern layer) with the least difference compared to the input impedance signature will result in a low value where a large difference between the input and categories (pattern layer) will result in a higher value. The output layer then identifies the structure thickness by choosing the node with the lowest value.

$$
P=f\left(x_{k}\right)=e^{\left(\sum_{k=1}^{N}\left(x_{k}-\left(x_{k}\right)_{T}\right)^{2} /\left(\sigma^{2} \cdot \sum_{k=1}^{N}\left(x_{k}\right)_{T}^{2}\right)\right)} .
$$

For the Gaussian function in (3), $x_{k}$ and $\left(x_{k}\right)_{T}$ represent the input data and the trained data, respectively. $\sigma$ of 0.5 is used for this study which is the constant controlling the width of the function requiring an educated guess based on the data. 
(1) Attach the PZT device to an impedance measurement

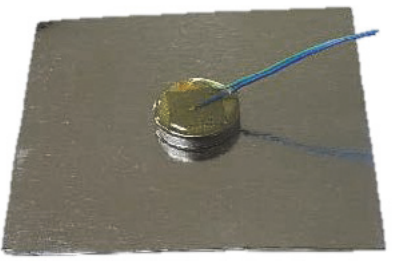

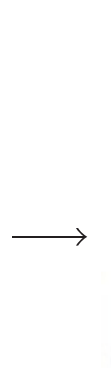
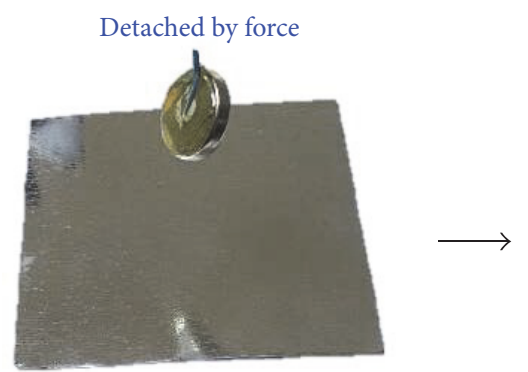

(3) PZT device reattached to another measurement

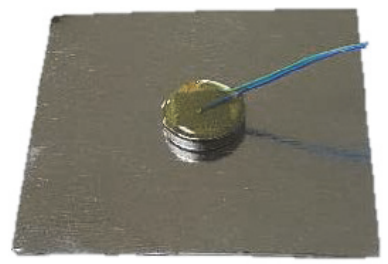

(2) Complete detachment of the PZT device

Figure 3: Attachment and reattachment of the PZT device.

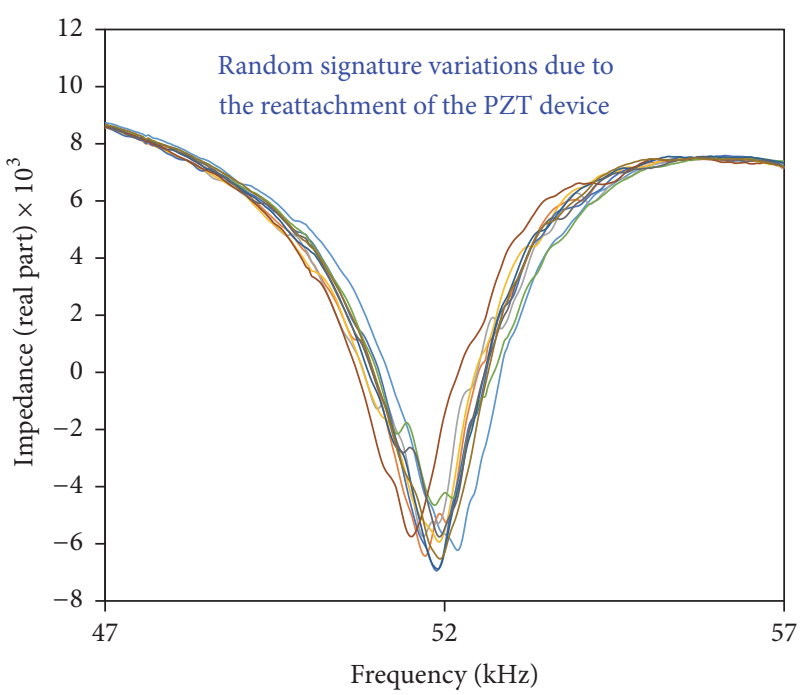

FIGURE 4: Impedance signatures from the reattachment test.

Once the training is complete, the PNN algorithm is ready for predicting the thickness of the host structure. Thus with a single measurement, it will predict the thickness of the target structure.

\section{Data Training and Experimental Setup}

5.1. Training the PNN Algorithm. The pattern layer of the PNN algorithm must be trained for it to correctly identify the thickness of a target structure. To achieve this, 10 impedance signatures are measured to be used as training data for each of the 5 categories. For the signature measurements, the PZT device is attached to the middle of a stack with 5 square metal plates $(100 \mathrm{~mm}$ length with either $0.15 \mathrm{~mm}$ or $0.3 \mathrm{~mm}$ thickness) where then the signature is measured. Afterwards, the PZT device is completely detached and attached again in the middle of the stack. This process is identical to the process shown in Figure 3 with the impedance signatures being measured until 10 signatures are acquired to be inserted into the first category as the training data (from nodes $P_{(5,1)}$ to $\left.P_{(5,10)}\right)$. The next set of training data is measured after removing a metal plate and then measuring the impedance signatures by attaching and detaching the PZT device until
10 signatures are obtained again (from nodes $P_{(4,1)}$ to $P_{(4,10)}$ ). Training is complete once the impedance signatures are measured with the last metal plate, totaling 50 impedance signatures to be used as the training data for nodes $P_{(5,1)}$ to $P_{(1,10)}$.

For attaching the PZT device to the middle of the metal plate, this was achieved manually to simulate a real field environment as it is virtually impossible to reattach the device to the exact same spot. Thus the presented study brings one step close for the EMI method to be used for practical applications. Figure 6 shows the impedance signatures measured for the training data used for the next section (Section 5.2) using a metal plate of $0.15 \mathrm{~mm}$ thickness. Figures 6(a)-6(e) shows 10 signature measurements from a single layer to 5 layers of metal plates and Figure 6(f) shows all the signatures combined to illustrate the overall trend of the impedance signatures. By observation, Figure 6(a) shows impedance signature variations mainly in the resonance frequency range between $52 \mathrm{kHz}$ and $55 \mathrm{kHz}$ where the downward peak goes in the up or down direction and shifting in the left or right direction. Similar observations are made for the rest of the figures from Figures 6(b)-6(e) where the impedance signature changes are focused in the resonance frequency region. Another observation made here is that the impedance signatures from Figure 6(e) seem to change more severely compared to Figure 6(a) with the existence of multiple small peaks. In addition, the downward peak for Figure 6(e) has decreased further down compared to Figure 6(a) to around 8000 ohms. Such reason can be due to the loss in metal thickness causing the weight to be lost where the structure becomes more prone to a higher level amplitude excitation. In Figure 6(f), combining all 50 impedance signatures shows a clear trend where the loss in the thickness causes the signature to shift in the left direction with the peaks decreasing towards the downward direction in general. Thus with these 50 pieces of training data inserted into the pattern layer of the PNN algorithm, it is now set to predict the thickness of the metal stack introduced in the following subsection.

5.2. Thickness Prediction Experiment Using $0.15 \mathrm{~mm}$ Thick Metal Plates. To predict the thickness of a metal stack subjected to thickness loss, 5 square metal plates with $100 \mathrm{~mm}$ length and $0.15 \mathrm{~mm}$ thickness are used. With this approach, one can reduce the thickness of the target structure with 


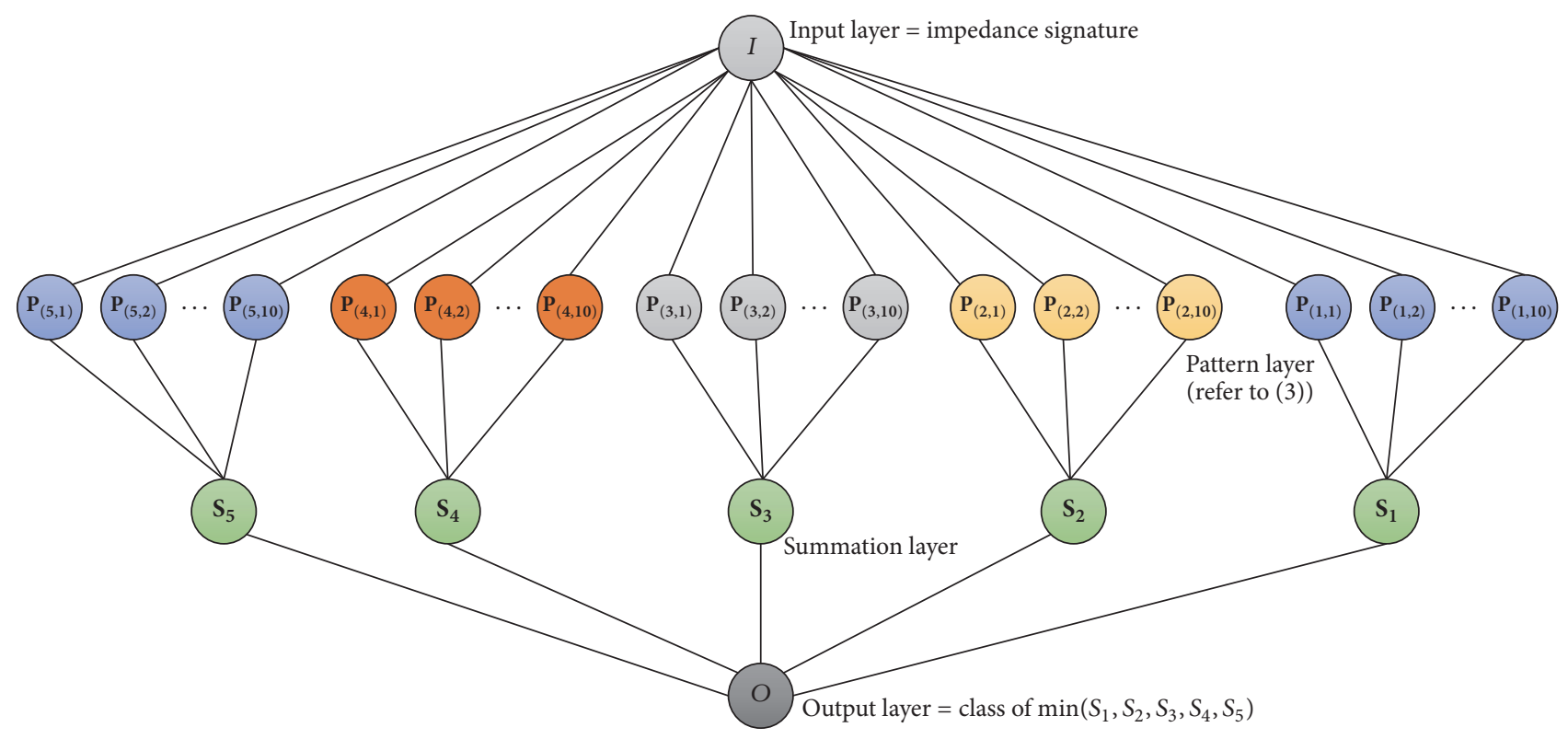

FIGURE 5: PNN algorithm created for the study.

an exact same thickness each time. Thus the 5 metals plates are stacked on top of one another and removing a single layer $(0.15 \mathrm{~mm})$ is defined as thickness loss damage. The PZT device is attached to the middle of the plate where the plates are held together by the magnetic force of the PZT device (Figure 7(a)). First, the impedance signature is measured with all 5 metal plates stacked together with the PZT device attached on top of the stack. This will be referred to as case " $0.15 T_{5} 5$ " where the acquired signature is inserted into the PNN algorithm for the thickness prediction of the metal stack. The next case, " $0.15 T_{-} 4$ " is achieved by removing a single plate from " $0.15 T_{-} 5$ " and then detaching and reattaching the PZT device, measuring impedance afterwards. Again, the acquired impedance signature is inserted into the PNN algorithm for a thickness prediction. The next case " $0.15 T_{\_} 3$ " is conducted by removing another plate and measuring the impedance where this process is continued until the impedance signature is measured for the last metal plate (cases " $0.15 T \_2$ " and " $0.15 T \_1$ "). Conducting these 5 test cases $\left(0.15 T_{-} 5\right.$ to $\left.0.15 T_{-} 1\right)$ will be referred to as "Test_\# $x$ " where $x$ represents the $n$th number of Test_\#. The reason for defining Test_\# is to check the reliability performance of the algorithm used in this study by repeating the experiment which is discuss in the later section. Figure 7(b) shows 5 signatures measured for $0.15 T_{\_} 5$ case where the variations in the signatures can be easily observed. Although reattaching the PZT device significantly changes the impedance signature, the PNN algorithm is assumed to correctly identify the thickness of the structure. Thus once the algorithm is equipped with enough training data, it becomes possible for one to use the reattachable PZT device to identify the thickness without any trail of sensor installation under the condition that magnets can be attached to the host structure.
5.3. Thickness Prediction Experiment Using $0.3 \mathrm{~mm}$ Thick Metal Plates. In this part of the experiment, 5 square metal plates of $100 \mathrm{~mm}$ in length and $0.3 \mathrm{~mm}$ thickness are used to conduct an experiment identical to the previous section. Again, the impedance signature is measured with all 5 metal plates stacked together as shown in Figure 8(a), referred to as case $0.3 T_{-} 5$ where the impedance signature is inserted into the input layer of the PNN algorithm for a thickness prediction. Then, impedance signature is measured after removing a metal plate until the last plate is remaining (cases $0.3 T_{-} 4$ to $0.3 T \_1$ ). For evaluating the repeatability performance of the proposed approach, 5 consecutive measurements are conducted by detaching and reattaching the PZT device for each of the 5 cases, totaling 25 impedance signatures. The 5 signature measurements for $0.3 T_{-} 5$ case can be seen in Figure 8(b) where the variations can be observed with peaks shifting in all 4 directions. After the completion of the proposed tests, the impedance signatures from Sections 5.2 and 5.3 are briefly compared to seek for differences in its trend with the use of the metal plates with different thicknesses.

\section{Results and Discussions}

6.1. Thickness Prediction of the $0.15 \mathrm{~mm}$ Metal Layer Stack. Table 2 shows the results of the PNN algorithm from the $0.15 T \_5$ case in Section 5.2 where the 5 impedance signatures measured for this case were inserted into the algorithm. Each row in the table represents the number of metal layers (labeled " $x$ ") in the stack and the columns represent the calculated values for the pattern layer. The last column represents the summation layer obtained from summing all the values in each row. The italic number (output layer) in this column is the minimum value from the column as the PNN algorithm 


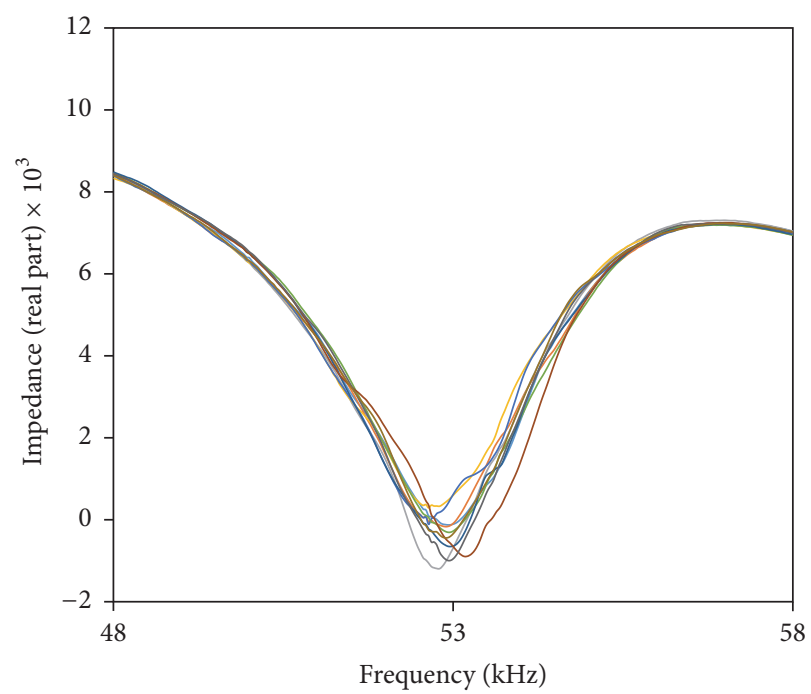

(a)

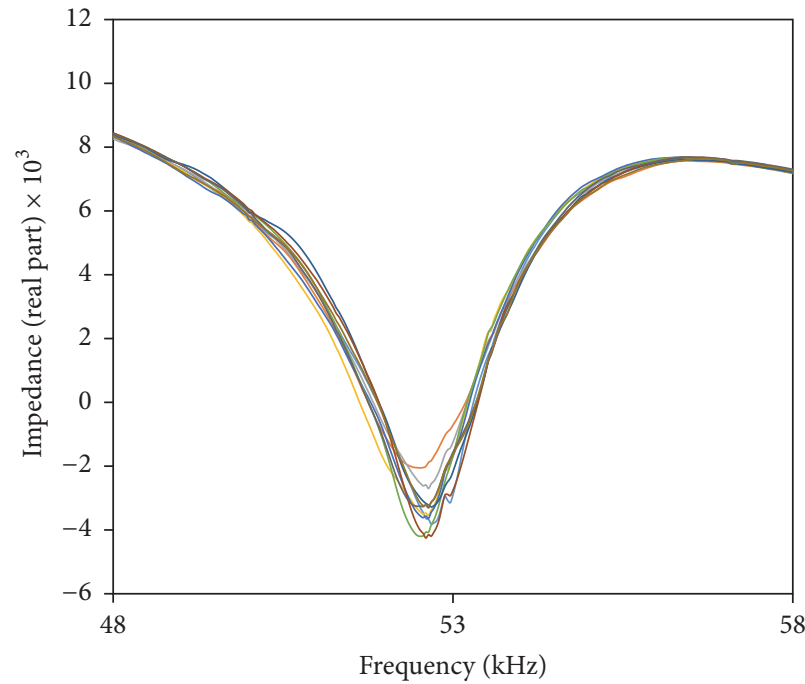

(c)

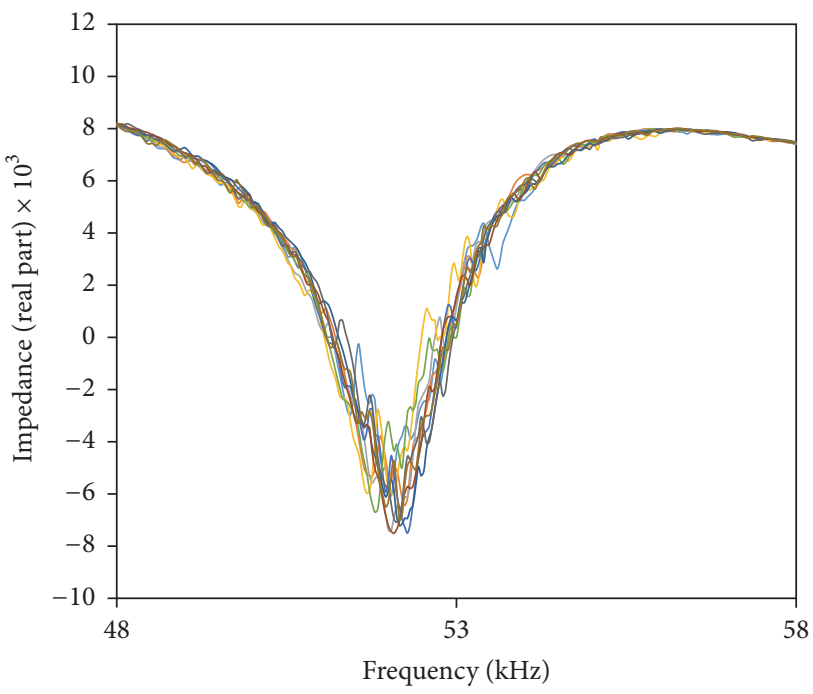

(e)

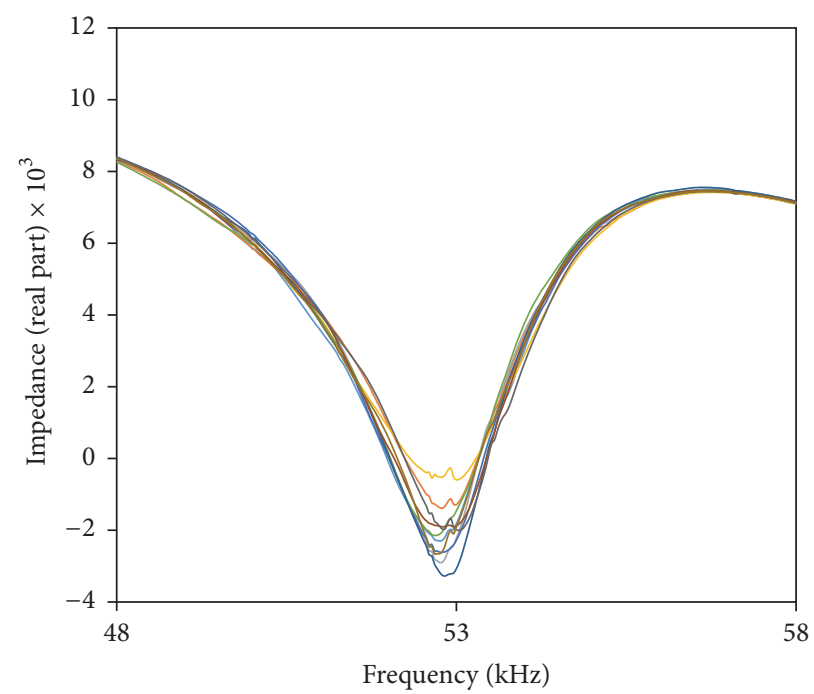

(b)

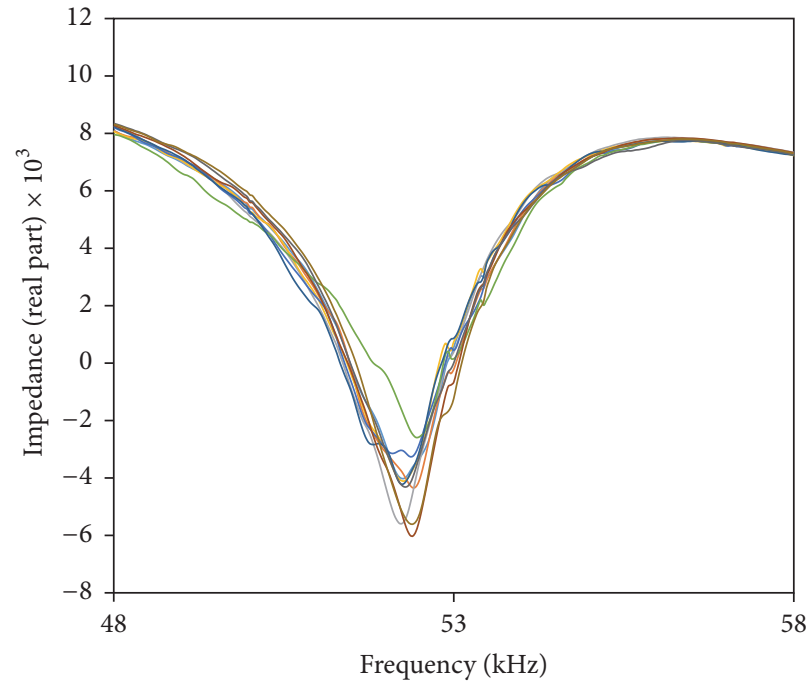

(d)

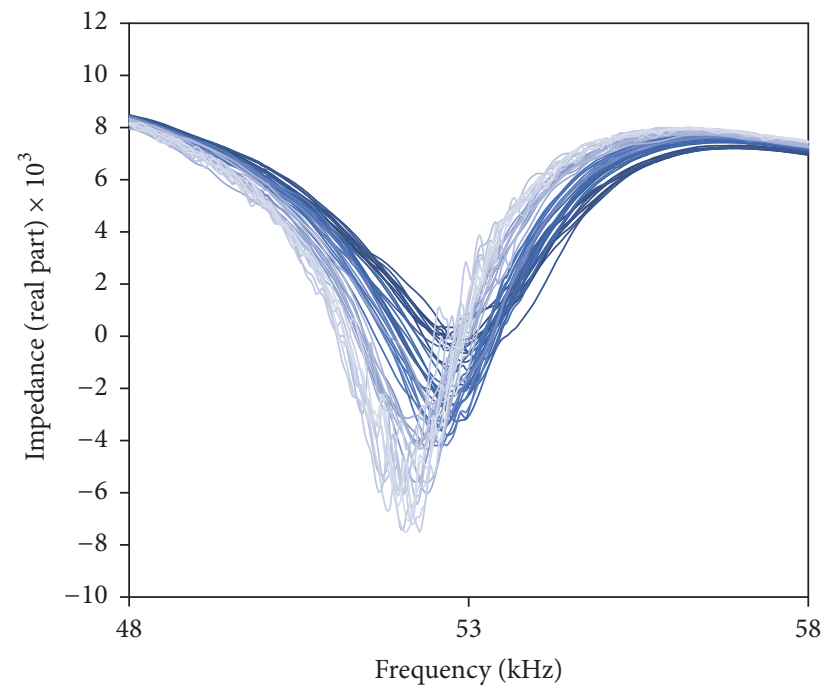

(f)

Figure 6: Impedance signatures of training data for (a) 5 metal plates, (b) 4 metal plates, (c) 3 metal plates, (d) 2 metal plates, (e) 1 metal plate, and (f) all results combined. 


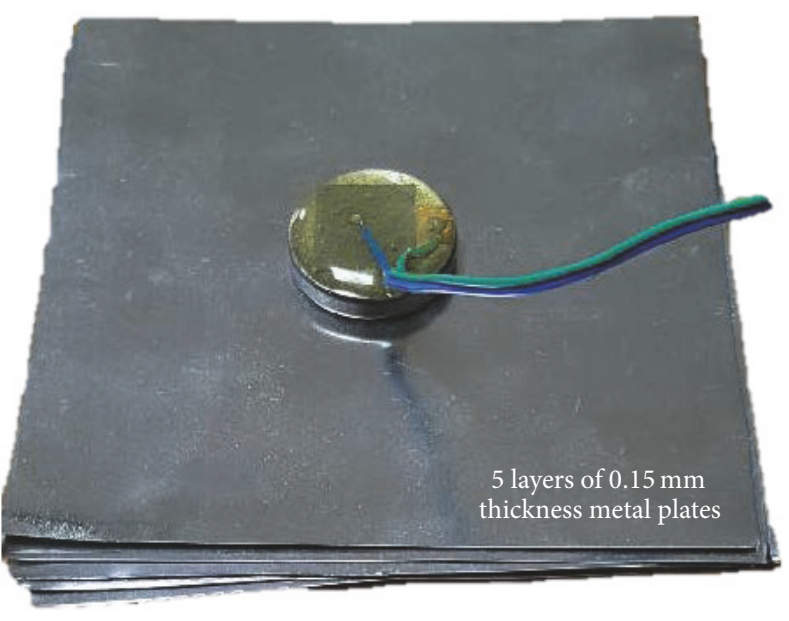

(a) 0.15_A case setup using the reattachable PZT device

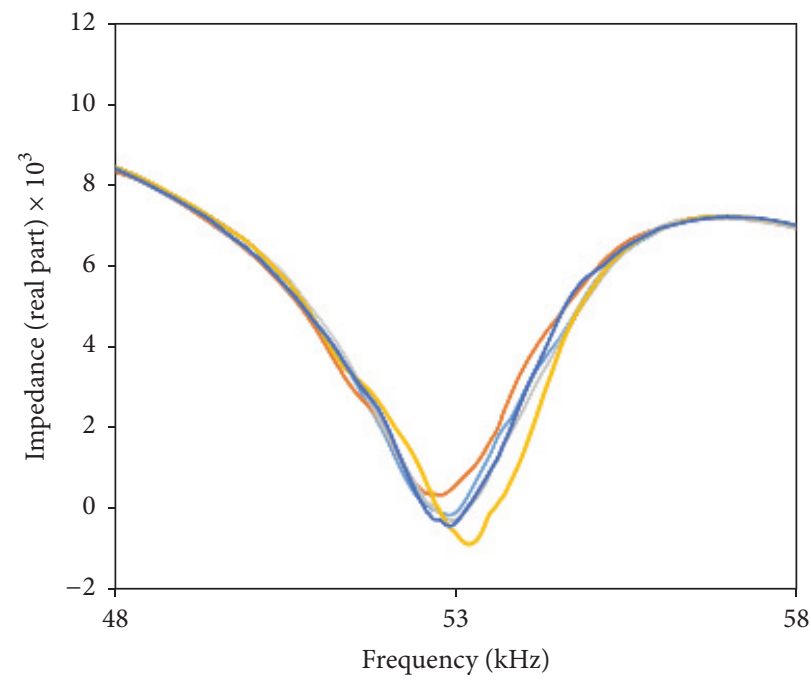

(b) 5 consecutive measurements for $0.15 \_$A case

Figure 7

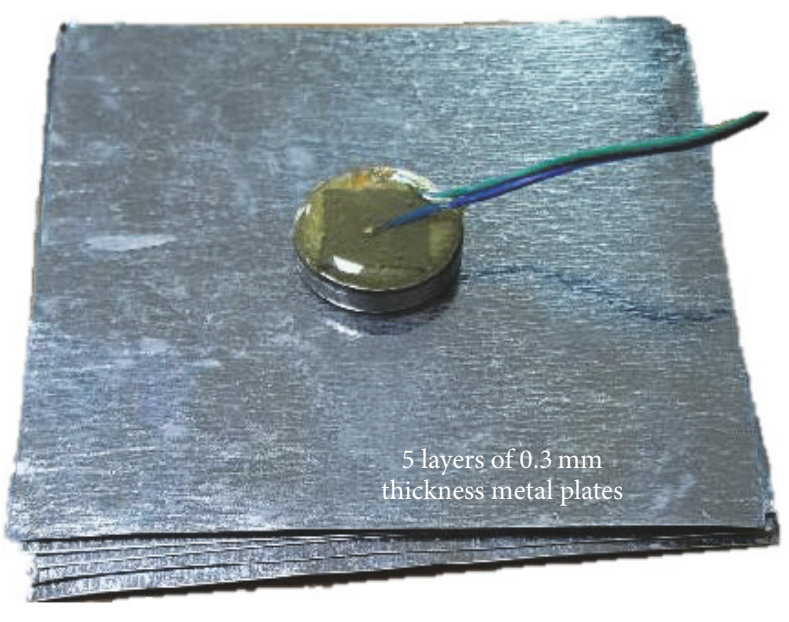

(a) 0.3_A case setup using the reattachable PZT device

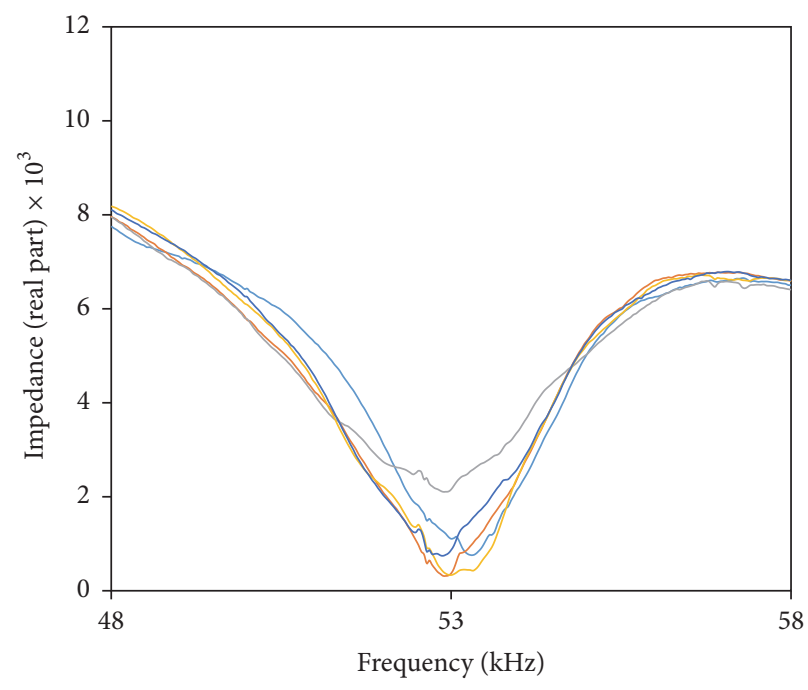

(b) 5 consecutive measurements for $0.3 \_A$ case

Figure 8

selects the minimum value and predicts that row as the " $x$ " number of metal layer(s) remaining from the stack. Thus with the first test, the italic number is 12.7, indicating that the stack has 5 metal layers, correctly identifying the status of the tested stack. Observing at the rest of the tests for 0.15T_5 case, the results (italic numbers) are 11.5, 11.4, 12.1, and 11.4 where a wrong prediction is made with the 12.1 value indicating that 4 metal layers are left in the stack. Out of the 5 tests from $0.15 T_{-} 5$ case, 4 predictions were correct showing $80 \%$ accuracy for this part of the test.

To identify the predictions made by the PNN algorithm for all the tests (Test_\#x for $0.15 T \_x$ cases), a bar graph is plotted in Figure 9. $y$-axis is the values obtained of the summation layer nodes of the PNN algorithm and $x$-axis is the test cases from $0.15 T \_5$ to $0.15 T_{\_}$. The numbers in the circle (5), (4), (3), (2), (1)) are the predicted number of plates remaining from the metal stack (the output layer value of the PNN algorithm). This is defined by selecting the lowest summation value from each case. Since Test_\#1 to Test_\#5 are conducted by detaching one plate at a time, it would be ideal for the PNN algorithm to predict the number of metal plates in the order of 5, 4, 3, 2, and 1 for each Test_\#x. This result can be seen for Test_\#1, Test_\#2, and Test_\#3 where Test_\#4 and Test_\#5 have one wrong prediction each $\left(0.15 T_{-} 5\right.$ and $\left.0.15 T \_2\right)$. Thus with the 25 output layer values from the 5 cases, 2 predictions were incorrect, resulting in 92\% (23/25) accuracy of the PNN algorithm.

6.2. Thickness Prediction of the $0.3 \mathrm{~mm}$ Metal Layer Stack. Figure 10 shows the bar plot for Test_\#x for $0.3 T \_x$ case 
TABLE 2: PNN algorithm results of case 0.15_A.

\begin{tabular}{|c|c|c|c|c|c|c|c|c|c|c|c|}
\hline & $P(x, 1)$ & $P(x, 2)$ & $P(x, 3)$ & $P(x, 4)$ & $P(x, 5)$ & $P(x, 6)$ & $P(x, 7)$ & $P(x, 8)$ & $P(x, 9)$ & $P(x, 10)$ & $\overline{S_{x}}$ \\
\hline & \multicolumn{11}{|c|}{ First measurement } \\
\hline$x=5$ & 1.2 & 1.3 & 1.3 & 1.4 & 1.4 & 1.2 & 1.2 & 1.2 & 1.2 & 1.3 & 12.7 \\
\hline$x=4$ & 1.5 & 1.4 & 1.5 & 1.3 & 1.5 & 1.5 & 1.6 & 1.4 & 1.3 & 1.5 & 14.5 \\
\hline$x=3$ & 1.8 & 1.8 & 1.8 & 2.1 & 2.0 & 2.1 & 1.7 & 1.8 & 1.9 & 1.7 & 18.7 \\
\hline$x=2$ & 2.6 & 2.6 & 3.0 & 2.8 & 2.6 & 2.1 & 2.9 & 2.9 & 2.5 & 2.6 & 26.6 \\
\hline \multirow[t]{2}{*}{$x=1$} & 3.3 & 3.7 & 4.0 & 3.8 & 3.8 & 3.6 & 3.7 & 3.9 & 3.5 & 3.7 & 37.0 \\
\hline & \multicolumn{11}{|c|}{ Second measurement } \\
\hline$x=5$ & 1.1 & 1.2 & 1.1 & 1.3 & 1.2 & 1.1 & 1.1 & 1.2 & 1.1 & 1.1 & 11.5 \\
\hline$x=4$ & 1.4 & 1.2 & 1.4 & 1.2 & 1.4 & 1.4 & 1.5 & 1.3 & 1.2 & 1.4 & 13.4 \\
\hline$x=3$ & 1.7 & 1.6 & 1.6 & 1.9 & 1.8 & 1.9 & 1.6 & 1.7 & 1.7 & 1.6 & 17.1 \\
\hline$x=2$ & 2.4 & 2.4 & 2.8 & 2.5 & 2.4 & 1.9 & 2.6 & 2.6 & 2.3 & 2.4 & 24.3 \\
\hline \multirow[t]{2}{*}{$x=1$} & 3.1 & 3.4 & 3.7 & 3.6 & 3.5 & 3.3 & 3.4 & 3.6 & 3.2 & 3.4 & 34.2 \\
\hline & \multicolumn{11}{|c|}{ Third measurement } \\
\hline$x=5$ & 1.1 & 1.1 & 1.2 & 1.2 & 1.2 & 1.1 & 1.1 & 1.2 & 1.1 & 1.1 & 11.4 \\
\hline$x=4$ & 1.5 & 1.3 & 1.5 & 1.2 & 1.5 & 1.5 & 1.6 & 1.5 & 1.4 & 1.5 & 14.5 \\
\hline$x=3$ & 1.8 & 1.7 & 1.7 & 2.0 & 1.9 & 2.0 & 1.7 & 1.9 & 1.9 & 1.7 & 18.3 \\
\hline$x=2$ & 2.4 & 2.5 & 2.8 & 2.5 & 2.4 & 1.9 & 2.7 & 2.7 & 2.4 & 2.5 & 24.8 \\
\hline \multirow[t]{2}{*}{$x=1$} & 3.1 & 3.5 & 3.7 & 3.6 & 3.5 & 3.4 & 3.5 & 3.6 & 3.3 & 3.4 & 34.6 \\
\hline & \multicolumn{11}{|c|}{ Fourth measurement } \\
\hline$x=5$ & 1.3 & 1.3 & 1.2 & 1.4 & 1.3 & 1.3 & 1.2 & 1.4 & 1.2 & 1.3 & 12.9 \\
\hline$x=4$ & 1.2 & 1.2 & 1.2 & 1.2 & 1.2 & 1.2 & 1.3 & 1.2 & 1.2 & 1.2 & 12.1 \\
\hline$x=3$ & 1.5 & 1.4 & 1.4 & 1.7 & 1.6 & 1.6 & 1.4 & 1.5 & 1.5 & 1.4 & 15.0 \\
\hline$x=2$ & 2.1 & 2.1 & 2.4 & 2.2 & 2.1 & 1.7 & 2.3 & 2.3 & 2.0 & 2.1 & 21.3 \\
\hline \multirow[t]{2}{*}{$x=1$} & 2.7 & 3.0 & 3.2 & 3.2 & 3.0 & 2.9 & 2.9 & 3.1 & 2.8 & 2.9 & 29.7 \\
\hline & \multicolumn{11}{|c|}{ Fifth measurement } \\
\hline$x=5$ & 1.1 & 1.1 & 1.2 & 1.1 & 1.1 & 1.1 & 1.1 & 1.3 & 1.2 & 1.1 & 11.4 \\
\hline$x=4$ & 1.6 & 1.3 & 1.6 & 1.3 & 1.6 & 1.5 & 1.7 & 1.5 & 1.4 & 1.5 & 15.0 \\
\hline$x=3$ & 1.9 & 1.7 & 1.7 & 2.1 & 2.0 & 2.0 & 1.8 & 1.9 & 1.9 & 1.8 & 18.8 \\
\hline$x=2$ & 2.4 & 2.5 & 2.8 & 2.5 & 2.4 & 1.9 & 2.6 & 2.7 & 2.3 & 2.5 & 24.6 \\
\hline$x=1$ & 3.0 & 3.4 & 3.7 & 3.5 & 3.5 & 3.3 & 3.5 & 3.6 & 3.3 & 3.4 & 34.2 \\
\hline
\end{tabular}

experiments where the numbers in the circle represent the number of metal layers left in the stack (output layer values). Again, it would be ideal to predict the number of plates decreasing from 5 to 1 for each Test_\#x. Here, only Test_\#4 and Test_\#5 have correctly predicted all the results with one wrong prediction resulting from Test_\#1 to Test_\#3. Thus with the 25 output layer values from the 5 cases, 3 predictions were incorrect, resulting in $88 \%(22 / 25)$ accuracy of the PNN algorithm.

To investigate the impedance signatures for the metal stack used in the previous section with the stack used in this section, Figure 11 is plotted using the 50 training data used for the PNN algorithm. In the figure, each of the impedance signatures is created by averaging 10 of the training data at each category. By observation, the amplitude of the peak in Figure 11(a) decreases within the range of 1000 2000 ohms each time the metal plate is removed with the signatures shifting in the left direction. Left shifting movement is also observed for Figure 11(b) where the peak decreases with much smaller amplitude for the top 4 impedance signatures. The last impedance signature representing a stack with one metal layer shows a large decrease in the amplitude. Comparing the two figures, a clear difference in the impedance signatures can be observed for the middle 3 signatures (stacks with 4, 3, and 2 metal layers) where the amplitudes are much smaller for Figure 11(b). One possible reason is due to the thicker metal layers $(0.3 \mathrm{~mm}$ versus $0.15 \mathrm{~mm})$ which affects the attachment strength as weaker magnetic force is experienced with increase in the distance. Since this study focusses on the possibility of reattaching the PZT device with the application of a neural network technique, a detailed investigation on the effect of magnetic attachment strength on impedance signatures will be dealt in a possible future work. An important fact here is that, regardless of the magnetic strength effect and the difference in the metal thickness, the proposed approach can successfully identify the thickness of the metal stack with high accuracy.

6.3. Improving the Accuracy of the PNN Algorithm. From the results of Sections 6.1 and 6.2, the accuracy of the PNN algorithm subjected to 5 cases tested were $92 \%$ and $88 \%$, respectively. Since each case involved measuring the 


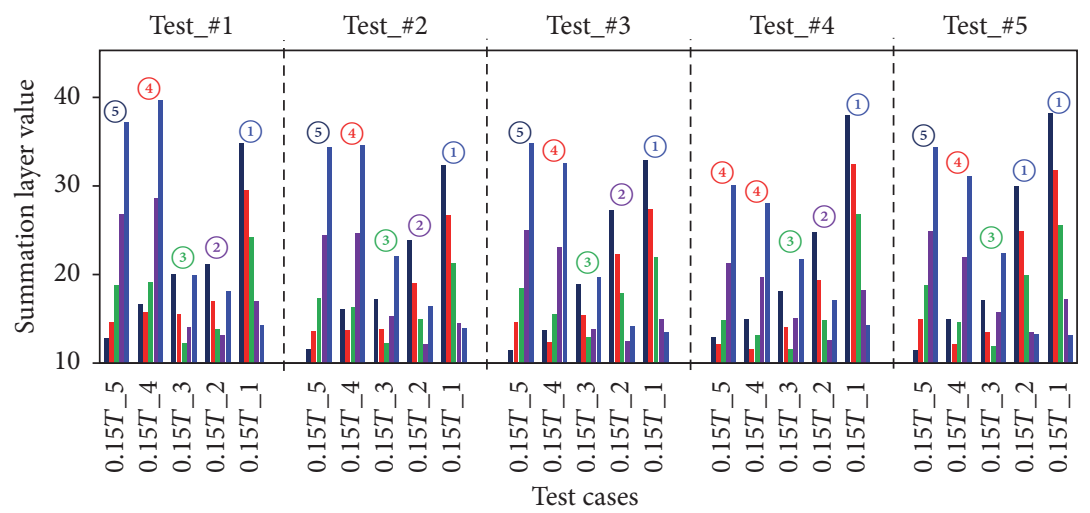

FiguRE 9: Summation and output layer results for Section 6.1.

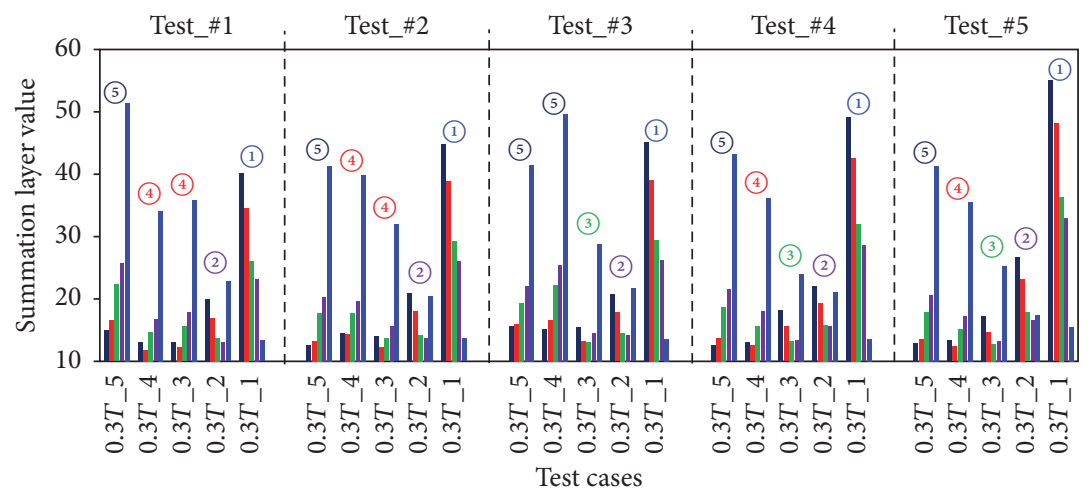

FIgURE 10: Summation and output layer results for Section 6.2.

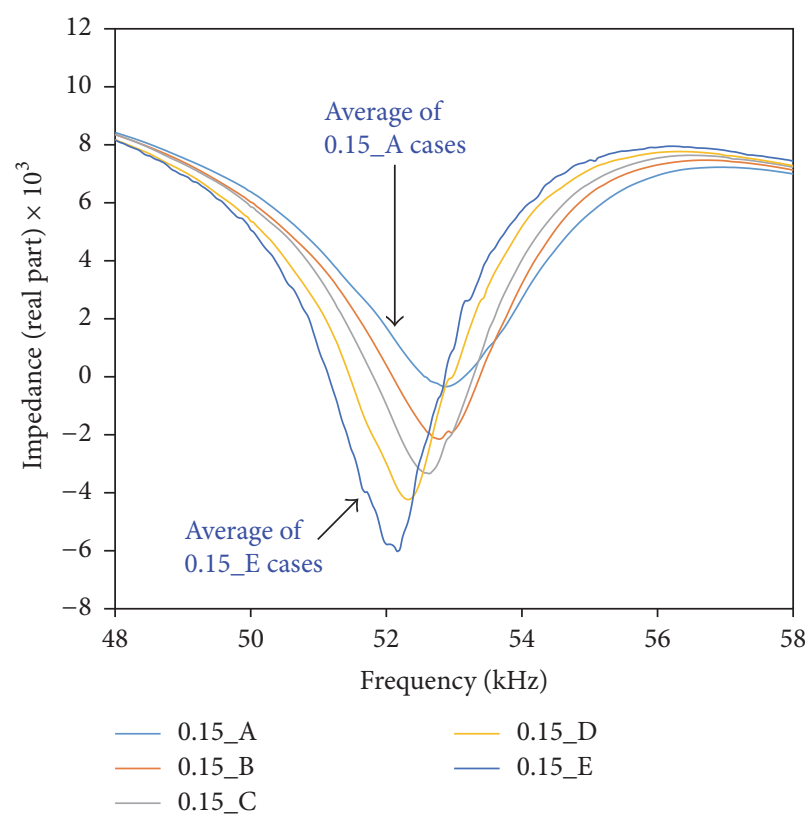

(a)

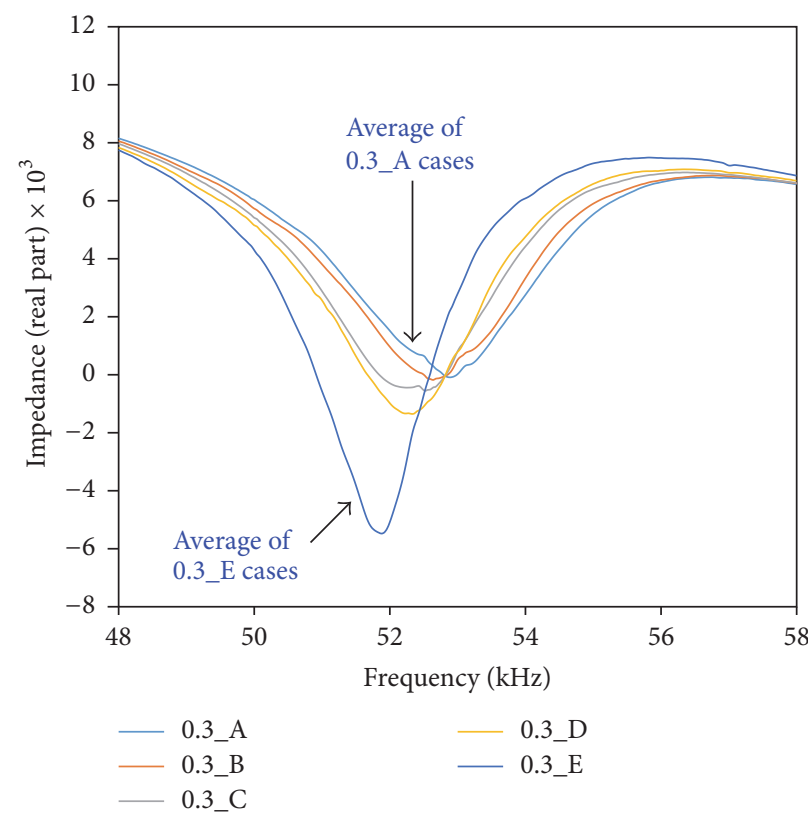

(b)

FIgURE 11: Averaged training data for (a) $0.15 \mathrm{~mm}$ thickness cases and (b) $0.3 \mathrm{~mm}$ thickness cases. 
impedance signature of the target structure 5 times, the method of improving the accuracy of the PNN algorithm can be easily achieved by selecting the output layer value that appears the most out of these 5 measurements. For an example, the $0.15 T \_5$ case resulted in 5 predictions where 4 of the predictions correctly identified the number of the metal layers in the stack (5 layers). Thus, out of the 5 measurements, choosing the most frequently appeared result (5 layers for this case) will result in improving the prediction accuracy of the PNN algorithm. By applying this approach, this results in $100 \%$ accurate prediction for all the cases $\left(0.15 T_{-} 5,0.15 T_{-} 4\right.$, $0.15 T_{\_} 3,0.15 T_{\_} 2,0.15 T_{-} 1,0.3 T \_5,0.3 T_{-} 4,0.3 T_{-} 3,0.3 T \_2$, and $\left.0.3 T \_1\right)$ conducted in this study.

\section{Conclusions}

In this study, a reattachable EMI method was used to identify the reduction in the metal layer thickness. Since the impedance signatures are highly sensitive subjected to any change in the property of a host structure, detaching and then reattaching the PZT transducer can significantly change the impedance signature. This can cause difficulty when identifying damage as the EMI method heavily relies on signature variations. Thus to overcome this problem, a neural network technique, PNN algorithm, was used to identify a stack of metal plates against reduction in thickness. The test was to evaluate the performance of the reattachable EMI method in conjunction with the PNN algorithm introduced in this study using two metal plate stacks with different thicknesses.

The first part of the test involved stacking 5 metal layers with $0.15 \mathrm{~mm}$ thickness where the reattachable PZT was attached in the middle of the stack for the experiment. Before the actual experiment, the PNN algorithm was trained, where the PZT device was attached and reattached 10 times while acquiring the impedance signatures. After the 10th measurement, a single layer from the metal stack was removed and another 10 impedance measurements were acquired until only one metal plate was left in the stack. 50 pieces of trained data were inserted into the pattern layer of the PNN algorithm to begin the experiment. Afterwards, Test_\#1 to Test_\#5 were conducted for the 5 cases $\left(0.15 T_{-} 5,0.15 T_{-} 4\right.$, $0.15 T \_3,0.15 T \_2$, and $\left.0.15 T \_1\right)$ where 25 output layer node values were obtained. Out of the 25 predictions, the PNN algorithm correctly predicted 23 outcomes, resulting in $92 \%$ accuracy of the test.

The second part of the test involved using 5 metal layers with an increased thickness of $0.3 \mathrm{~mm}$. Again, 50 pieces of training data were obtained and Test_\#1 to Test_\#5 were conducted for the 5 cases $\left(0.3 T_{-} 5,0.3 T_{-} 4,0.3 T_{-} 3\right.$, $0.3 T \_2$, and $\left.0.3 T \_1\right)$ where 25 output layer node values were obtained. Out of the 25 predictions, the PNN algorithm correctly predicted 22 outcomes, resulting in $88 \%$ accuracy of the test. Afterwards, 10 pieces of trained data measured at different metal layers were averaged for all cases to investigate any existence of trend between the two different thickness metal stacks where a clear difference in the signatures were observed. Although the amplitude of the impedance signatures decreased with each removal of the metal plate, the thicker stack $(0.3 \mathrm{~mm}$ layers $)$ experienced smaller decrease in the peak amplitude compared to the thinner stack ( $0.15 \mathrm{~mm}$ layers). Nevertheless, the introduced PNN algorithm successfully identified the thickness of the metal stack with high accuracy. For increasing the accuracy of the PNN algorithm introduced in this study, an approach was stated by selecting the outcome that appeared the most out of the 5 measurements for each of the cases. Since 5 impedance signatures were measured for each of the cases, selecting the most frequent results from the PNN algorithm resulted in $100 \%$ accuracy for both of the metal stacks.

\section{Future Work}

Bridges and buildings are usually inspected using NDT methods where the increase in complexity and height of the structure cause difficulty during inspection. Such work can be time-consuming and to overcome this problem, this work is to contribute towards a possibility of conducting an inspection using drones. One of the major disadvantages of a local method such as the EMI method is the small sensing range, and this could be solved by using a drone which can easily fly onto virtually any surfaces of a structure. A possible next step in this research would be to install an impedance measuring device onto a drone and conduct the reattachable EMI method against real damage scenarios. In addition, with the ability of the drones to be able to fly, a solar energy harvesting device will also be considered to create a drone that can self-recharge to create a system that requires zero energy input.

\section{Conflicts of Interest}

The authors declare that there are no conflicts of interest regarding the publication of this paper.

\section{Acknowledgments}

The research was supported by a grant from "Development of Infrastructure Technology for Hyper Speed Transportation System (20170095-001)" funded by Korea Institute of Civil Engineering and Building Technology (KICT), South Korea.

\section{References}

[1] C. Liang, F. P. Sun, and C. A. Rogers, "Coupled electro-mechanical analysis of adaptive material systems-determination of the actuator power consumption and system energy transfer," Journal of Intelligent Material Systems and Structures, vol. 5, no. 1, pp. 12-20, 1997.

[2] L. Yu, V. Giurgiutiu, and P. Pollock, "A multi-mode sensing system for corrosion detection using piezoelectric wafer active sensors," in Proceedings of the The 15th International Symposium on: Smart Structures and Materials \&amp; Nondestructive Evaluation and Health Monitoring, p. 69322H, 2008.

[3] V. Talakokula, S. Bhalla, R. J. Ball et al., "Diagnosis of carbonation induced corrosion initiation and progression in reinforced concrete structures using piezo-impedance transducers," Sensors and Actuators, A: Physical, vol. 242, pp. 79-91, 2016. 
[4] G. E. Simmers Jr., H. A. Sodano, G. Park, and D. J. Inman, "Detection of corrosion using piezoelectric impedance-based structural health monitoring," AIAA Journal, vol. 44, no. 11, pp. 2800-2803, 2006.

[5] S. Na, R. Tawie, and H.-K. Lee, "Electromechanical impedance method of fiber-reinforced plastic adhesive joints in corrosive environment using a reusable piezoelectric device," Journal of Intelligent Material Systems and Structures, vol. 23, no. 7, pp. 737747, 2012.

[6] W. S. Na, "Progressive damage detection using the reusable electromechanical impedance method for metal structures with a possibility of weight loss identification," Smart Materials and Structures, vol. 25, no. 5, Article ID 055039, 2016.

[7] G. Park, H. H. Cudney, and D. J. Inman, "Impedance-based health monitoring of civil structural components," Journal of Infrastructure Systems, vol. 6, no. 4, pp. 153-160, 2000.

[8] Y. Yang and Y. Hu, "Electromechanical impedance modeling of PZT transducers for health monitoring of cylindrical shell structures," Smart Materials and Structures, vol. 17, no. 1, Article ID 015005, 2008.

[9] R. Shanker, S. Bhalla, A. Gupta, and M. Praveen Kumar, "Dual use of PZT patches as sensors in global dynamic and local electromechanical impedance techniques for structural health monitoring," Journal of Intelligent Material Systems and Structures, vol. 22, no. 16, pp. 1841-1856, 2011.

[10] S. Na and H. K. Lee, "Neural network approach for damaged area location prediction of a composite plate using electromechanical impedance technique," Composites Science and Technology, vol. 88, pp. 62-68, 2013.

[11] G. Park, C. R. Farrar, F. L. Di Scalea, and S. Coccia, "Performance assessment and validation of piezoelectric activesensors in structural health monitoring," Smart Materials and Structures, vol. 15, no. 6, pp. 1673-1683, 2006.

[12] V. G. M. Annamdas and M. A. Radhika, "Electromechanical impedance of piezoelectric transducers for monitoring metallic and non-metallic structures: a review of wired, wireless and energy-harvesting methods," Journal of Intelligent Material Systems and Structures, vol. 24, no. 9, pp. 1021-1042, 2013.

[13] S. Na and H. K. Lee, "A multi-sensing electromechanical impedance method for non-destructive evaluation of metallic structures," Smart Materials and Structures, vol. 22, no. 9, Article ID 095011, 2013.

[14] F. P. Sun, Z. Chaudhry, C. Liang, and C. A. Rogers, "Truss structure integrity identification using PZT sensor-actuator," Journal of Intelligent Material Systems and Structures, vol. 6, no. 1, pp. 134-139, 1995.

[15] D. F. Specht, "Probabilistic neural networks," Neural Networks, vol. 3, no. 1, pp. 109-118, 1990. 


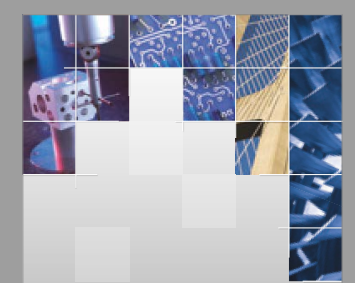

\section{Enfincering}
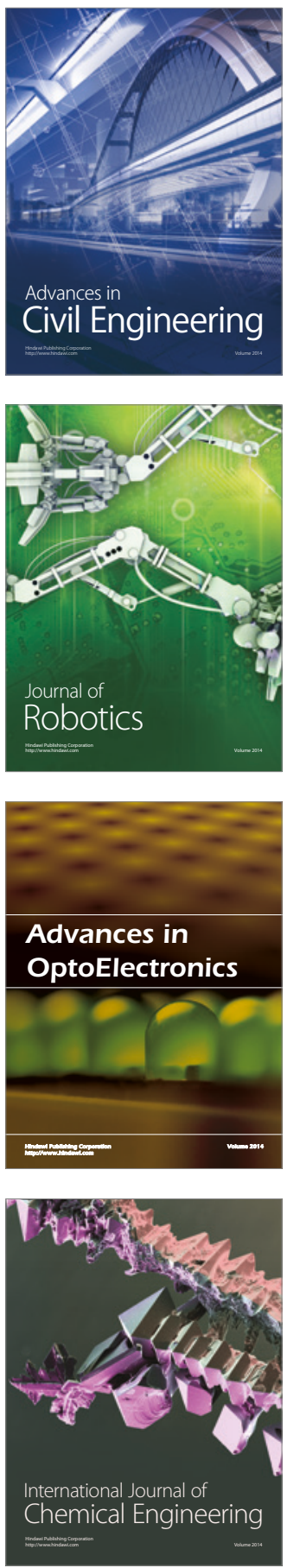

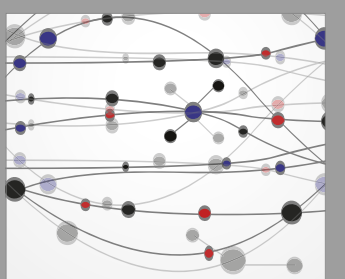

The Scientific World Journal

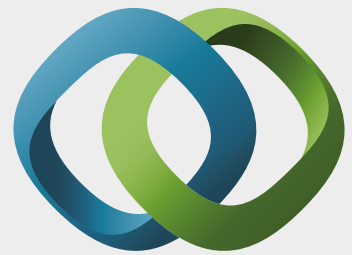

\section{Hindawi}

Submit your manuscripts at

https://www.hindawi.com
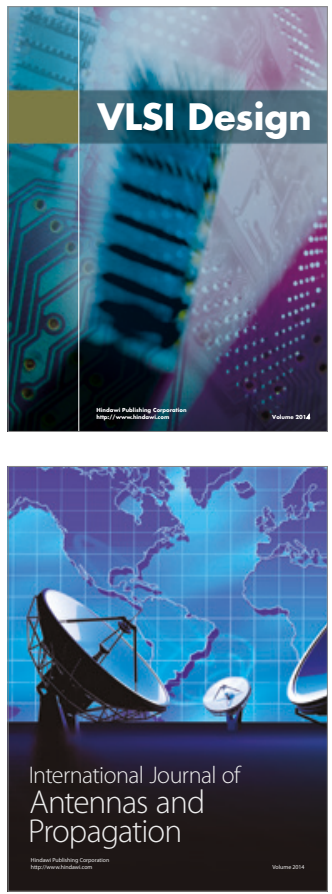

\section{Rotating}

Machinery
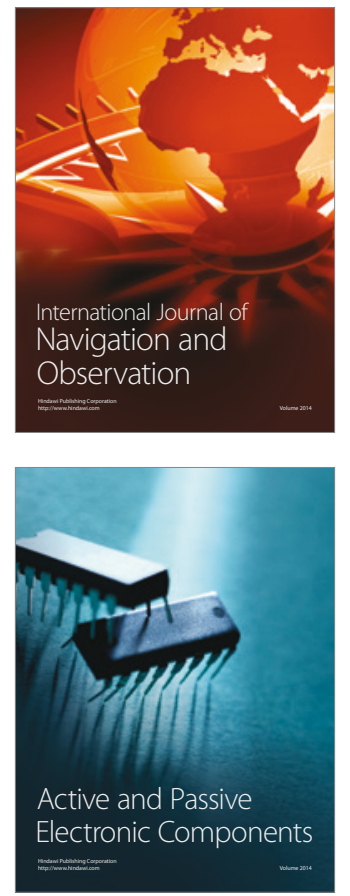
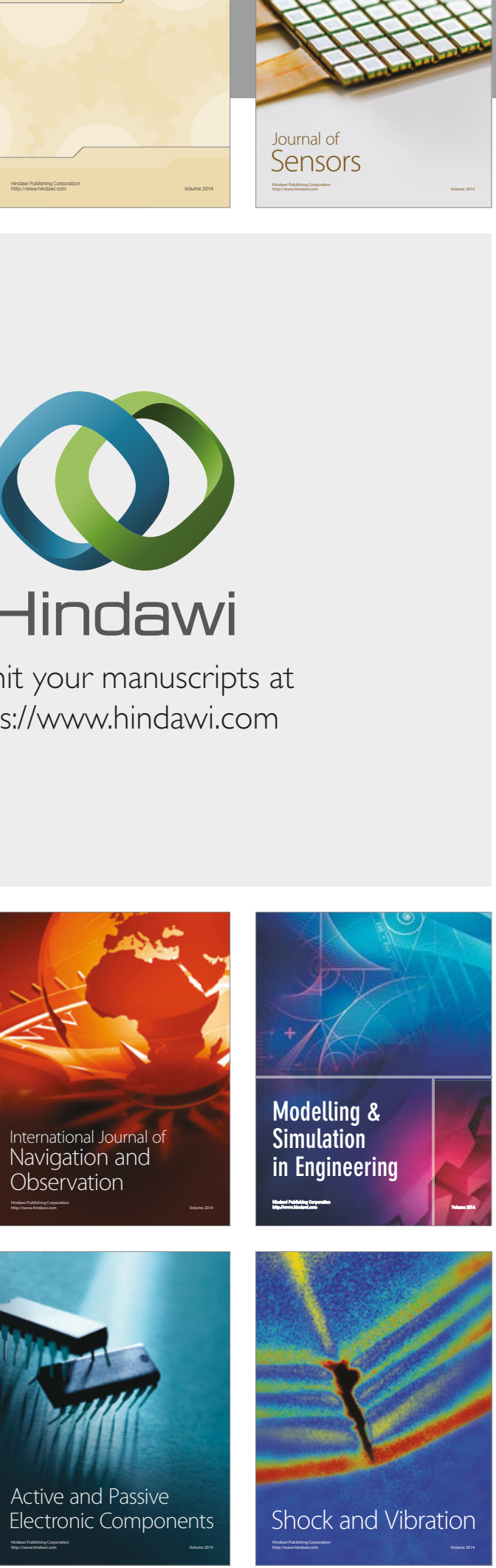
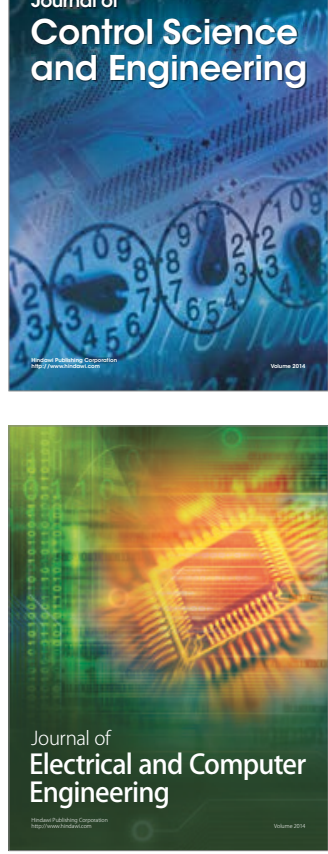

Distributed

Journal of

Control Science

and Engineering
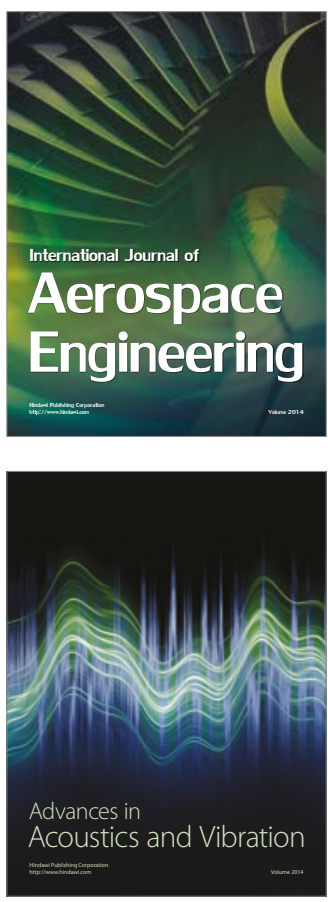

Sensor Networks 